\title{
Radio Resource Allocation for Low-Medium-Altitude Aerial Platform Based TD-LTE Networks against Disaster
}

\author{
Liqiang Zhao ", Jiangtao Yi ${ }^{*}$, Fumiyuki Adachi ${ }^{\dagger}$, Chi Zhang ${ }^{*}$, and Hailin Zhang ${ }^{*}$ \\ * State Key Laboratory of Integrated Services Networks, Xidian University, Xi'an, Shaanxi, China. \\ Department of Electrical and Communications Engineering, Tohoku University, Sendai, Japan. \\ E-mail: lqzhao@mail.xidian.edu.cn
}

\begin{abstract}
In order to provide an increased capacity for voice users in emergency scenarios, a low-medium-altitude aerial platform based time-division-duplex long term evolution (TD-LTE) system referred to as Aerial LTE, is presented in this paper. Furthermore, a novel radio resource allocation mechanism is proposed for both the Aerial LTE downlink and uplink, which is modeled as a cooperative game to provide real-time voice communications with as many users as possible. Our simulation results demonstrate that compared with the traditional radio resource management algorithms, the proposed algorithm achieves the largest capacity, i.e., the number of serviced users.
\end{abstract}

Keywords- Low Medium-Altitude Aerial Platform, TD-LTE, Radio Resource Allocation, Capacity, Game Theory

\section{INTRODUCTION}

On 12 May 2008, Wenchuan in China was hit by a destructive earthquake. All the terrestrial telecommunication systems were damaged. Hence Wenchuan lost its links with the outside world. A similar scenario occurred after the recent tsunami near Sendai, Japan. Since then low-medium-altitude aerial platforms (LMAPs) as emergency communication systems, have attracted significant interest from government, academia and industry [1]. Captive balloons are stationary at low-medium-altitudes ranging from about $2 \mathrm{~km}$ to $20 \mathrm{~km}$ and cover a wide area. This makes them an attractive option for carrying telecommunication equipment. LMAPs may carry equipment obeying diverse wireless standards, including 3G/B3G, LTE, WiMAX or combinations of them. The time-division-duplex long term evolution (TD-LTE) system is considered as one of the candidate technologies for the next generation mobile communications systems [2]. Hence an aerial platform-based TD-LTE system, Aerial LTE, is presented in this paper for providing emergency services in disaster areas.

One of the key technologies designed for managing a high tele-traffic during disasters is radio resource management (RRM). Diverse RRM algorithms have been proposed for LTE to achieve different requirements. For example, the authors of [3] and [4] proposed algorithms for maximizing the system throughput, while ensuring fairness in both the LTE downlink (DL) and uplink (UL). A joint resource allocation algorithm simultaneously considering the time-frequency-power-domain based on the Nash bargaining solution (NBS) was implemented in a multi-cell WiMAX-based LMAP system in our previous work [1]. Moreover, providing seamless power supply for LMAPs is a challenging problem, especially in disaster areas. The authors of [5-8] have adaptively allocated the power to subcarriers, but they have not considered fairness in the context of diverse applications.

However, all the above algorithms did not consider the relevant context of emergency communications. Facing disaster, people want to talk with their families, friends, etc, in order to confirm their safety. So the most important requirement is to provide real-time voice communications with as many users as possible, where quality of service (QoS), e.g., short delay and broadband, is not a serious problem [14]. In this study we use game theory for the RRM algorithms with high capacity, i.e., the number of serviced voice users, in the context of the Aerial LTE uplink and downlink.

The rest of this paper is organized as follows. In Section II we develop the system model of both the Aerial LTE UL and DL. In Section III and IV, a game-theoretic radio resource allocation algorithm with high capacity is detailed. In Section V, simulations are carried out to evaluate the performance of the proposed algorithm. Our conclusions are offered in Section VI.

\section{System ModeL}

A typical Aerial LTE scenario includes the aerial NodeBs (aNBs) and the user equipments (UEs) on the ground. Each aNB supports a traffic cell and all the UEs are randomly distributed in these areas. According to the LTE standard [2], our system relies on orthogonal frequency diversion multiplexing (OFDM) in the DL and single carrier frequency division multiple access (SC-FDMA) in the UL. The scheduler of an aNB is responsible for allocating radio resources to both the UL shared channel (UL-SCH) and to the DL shared channel (DL-SCH), in order to maximize the bandwidth or power efficiency, while ensuring fairness among the UEs as well as

This work is supported by the 111 Project (B08038), State Key Laboratory of Integrated Services Networks (ISN090105), Program for New Century Excellent Talents in University (NCET-08-0810), National Natural Science Foundation of China (No. 60772137), and UK-China Science Bridges: R\&D on (B)4G Wireless Mobile Communications. 
maintaining the required QoS for different traffic requirements. The radio resources defined in the LTE standard include the resource blocks (RB), the specific modulation and coding schemes (MCS), the power allocation schemes and the antenna options. In other words, we have five resource categories which are the time, frequency, code, power, and spatial domains respectively. A RB, which is composed of 12 consecutive sub-carriers, occupying $180 \mathrm{kHz}$ in the frequency domain (FD) and one time slot of $0.5 \mathrm{~ms}$ duration in the time domain (TD), is the minimum frequency-time resource unit. A time slot $\left(T_{\text {slot }}\right)$ hosts 6 or 7 OFDM symbols in the DL or SC-FDMA symbols in the UL. This allows us to formulate the RB allocation in a matrix-like structure. However, in line with other standards, the LTE standards do not explicitly specify the RRM schemes because different service-providers and operators have different spectrum allocations. Furthermore, this open structure facilities the creation of new innovative algorithms. For simplicity, we only consider RB and power allocation in this paper.

There are many bandwidth- or power-efficient RRM algorithms [3-8]. However, to our best knowledge, no one has considered how to maximize the number of serviced voice users, or referred as capacity or user efficiency in this paper. Hence, first of all, we should present a novel utility function for high capacity.

The utility of the $n$-th $\mathrm{UE}\left(\mathrm{UE}_{n}\right)$ is defined as $U_{n}\left(\gamma_{n}\right)=r_{n}\left(\gamma_{n}\right) / r_{n, \max }$, where $r_{n}$ is its attainable data rate and $r_{n, \max }$ is its maximum required data rate. The user utility is the function of its signal to interference plus noise ratio (SINR), $\gamma_{n}$. And the system utility, $\sum_{n=1}^{N} U_{n}\left(\gamma_{n}\right)=\sum_{n=1}^{N} \frac{r_{n}\left(\gamma_{n}\right)}{r_{n, \max }}$, where $N$ is the number of UEs in a given cell. Obviously, the utility of $\mathrm{UE}_{n}$ ranges between 0 and 1 , and $\mathrm{UE}_{n}$ benefits from an increased utility as it approaches 1 . Hence, the more voice users are serviced, the larger system's utility is. In other words, the system's utility indicates capacity, or the number of serviced voice users.

Hence, the proposed RRM with high capacity can be modeled as the following optimization problem with constraints

$$
\max _{\mathbf{X}, \mathbf{P}} \sum_{n=1}^{N} U_{n}\left(\gamma_{n}\right)=\max _{\mathbf{X}, \mathbf{P}} \sum_{n=1}^{N} \frac{r_{n}\left(\gamma_{n}\right)}{r_{n, \max }}
$$

subject to:

$$
\begin{gathered}
P_{n, k, t} \geq 0 \quad \forall n, k, t \\
\sum_{n=1}^{N} x_{n, k, t}=1 \quad x_{n, k, t}=\{0,1\} \quad \forall k, t \\
\sum_{n} \sum_{k} \sum_{t} P_{n, k, t} \leq P_{\max , a N B},
\end{gathered}
$$

where $\mathbf{X}=\left\{x_{n, k, t}\right\}$ and $\mathbf{P}=\left\{P_{n, k, t}\right\}$ denote the RB allocation (RBA) and power allocation (PA) matrix, respectively. If $R B_{k, t}$ at the $k$-th row and $t$-th column of resource grid is assigned to $\mathrm{UE}_{n}$, then we have $x_{n, k, t}=1$ and its assigned power is $P_{n, k, t}$; otherwise, we have $x_{\mathrm{n}, \mathrm{k}, \mathrm{t}}=0$. Since a RB in each aerial NodeB can be assigned to one and only one UE, we have $\sum_{n=1}^{N} x_{n, k, t}=1$. Furthermore, the sum of the assigned transmission power is less than the maximum transmission power $P_{\max , a N B}$ of aNBs.

\section{USER-EFFICIENT RADIO RESOURCE ALLOCATION FOR THE DOWNLINK}

Since all UEs compete with each other for the time-frequency-power resources, cooperative game theory is adopted for optimizing RRM for the DL. However, the optimization problem in (1) is an NP-hard combinatorial problem. In order to reduce the complexity imposed, we partition the optimization problem into two sub-problems having a lower complexity, namely into separate time-frequency resource allocation and power allocation.

\section{A. Time-frequency resource allocation}

It is assumed that the transmit power will be equally distributed among RBs, i.e., we have

$$
P_{n, k, t}=\left\{\begin{array}{c}
P_{\max , a N B} /(K \cdot T) \quad x_{n, k, t}=1 \\
0 \quad x_{n, k, t}=0
\end{array} .\right.
$$

where $\mathrm{K}$ and $\mathrm{T}$ denote the number of rows and columns of the time-frequency resource grid, respectively.

Given the power allocation matrix $\mathrm{P}$, the optimization problem (1) may be simplified to

$$
\max _{\mathbf{X}} \sum_{n=1}^{N} U_{n}\left(\gamma_{n}\right)=\max _{\mathbf{X}} \sum_{n=1}^{N} \frac{r_{n}\left(\gamma_{n}\right)}{r_{n, \max }}
$$

subject to: $\quad \sum_{n=1}^{N} x_{n, k, t}=1 \quad x_{n, k, t}=\{0,1\} \quad \forall k, t$,

By using the classic Lagrange multiplier method [1], we arrive at

$$
\left\{\begin{array}{ll}
U_{n}^{\prime}\left(\gamma_{n}\right) \times r_{n}=\lambda & \text { if } x_{n, k, t}=1 \\
U_{n}^{\prime}\left(\gamma_{n}\right) \times r_{n} \leq \lambda & \text { if } x_{n, k, t}=0
\end{array} .\right.
$$

Thus the closed form solution of the RB assignment can be obtained in the form of

$$
n=\underset{n}{\arg \max }\left[U_{n}^{\prime}\left(\gamma_{n}\right) \times r_{n}\right],
$$

where $\mathrm{RBk}, \mathrm{t}$ is allocated to the nth UE for maximizing $U_{n}^{\prime}\left(\gamma_{n}\right) \times r_{n}$. 


\section{B. Power allocation}

So far we have addressed the RB allocation problem under the assumption that the same power is assigned to all RBs. Therefore, based on the generated RBA results, we simply have to re-allocate the transmission power among all the RBs. The optimization problem (1) can hence be re-formulated as:

$$
\max _{\mathbf{P}} \sum_{n=1}^{N} U_{n}\left(\gamma_{n}\right)=\max _{\mathbf{P}} \sum_{n=1}^{N}\left(\frac{1}{r_{n, \max }} \cdot \sum_{k=1}^{K} x_{n, k, t} r_{n, k, t}\right)
$$

subject to:

$$
\begin{aligned}
& P_{n} \geq 0 \quad \forall n \\
& \sum_{n=1}^{N} P_{n} \leq P_{\max , a N B} .
\end{aligned}
$$

Similarly, after using the Lagrange multiplier method, we take the derivatives of $U_{n}\left(\gamma_{n}\right)$ with respect to $P_{n}$. Then, according to the Karush-Kuhn-Tucker (KKT) condition [11], we arrive at

$$
\left\{\begin{array}{c}
U_{n}^{\prime}\left(\gamma_{n}\right) \cdot \gamma_{n}^{\prime}\left(P_{n}\right)-\lambda=0 \\
\sum_{n=1}^{N} P_{n} \leq P_{\max , a N B}
\end{array} .\right.
$$

By solving the above equations, we finally arrive at the optimal power allocation matrix $\mathbf{P}$.

\section{Joint time-frequency-power resource allocation}

To summarize, the proposed algorithm may be decomposed into three steps, as shown in Fig. 1. Firstly, we assume that the transmission power is equally distributed among all the RBs. Based on the estimated SINR, the optimal RB assignment matrix $\mathbf{X}$ may be obtained according to Equation (7). Secondly, based on the RB allocation results, the power allocation matrix $\mathbf{P}$ can be generated according to Equation (10). Finally, we substitute the power allocation results obtained during the second step into the first step and update the RB assignment. This iteration continues until the result converges.

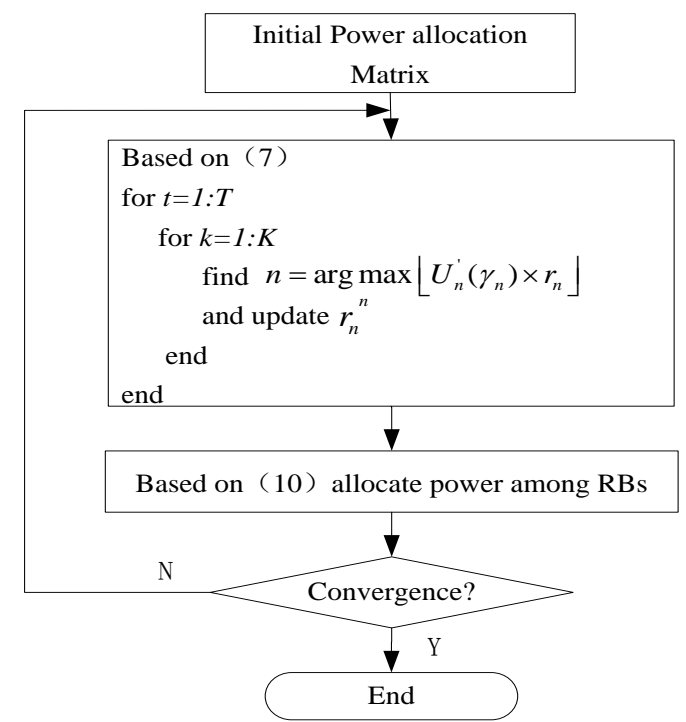

Figure 1. Joint time-frequency-power resource allocation

\section{USER-EFFICIENT RADIO RESOURCE ALLOCATION FOR THE UPLINK}

Compared to the UL, typically, more data are transmitted in the DL. However, the UL may occupy most of the radio resources in emergency communications. A short message may be transmitted in the DL to inform rescuers about their expected actions. On the other hand, large amounts of information such as statistical data, images and video may be transmitted in the UL. Hence, a user-efficient RRM algorithm may be formulated for the UL, which is modeled as:

$$
\max _{\mathbf{P}} U_{n}\left(\gamma_{n}\right)=\max _{\mathbf{P}} \frac{r_{n}}{r_{n, \max }}
$$

subject to:

$$
0 \leq P_{n} \leq P_{\max , U E} \quad \forall n,
$$

where the maximum transmission power of UEs is $P_{\text {max,UE }}$.

It has been shown that the above utility function $U_{n}\left(\gamma_{n}\right)$ is quasi-concave around the value of $P_{n}$ [10]. Hence, after taking the derivatives of $U_{n}\left(\gamma_{n}\right)$ with respect to $P_{n}$, and setting the result to 0 , we arrive at the optimal solution as:

$$
P_{n}^{\Delta}=\frac{f\left(\gamma_{n}\right)}{f^{\prime}\left(\gamma_{n}\right) \cdot \gamma_{n}^{\prime}},
$$

where $f\left(\gamma_{n}\right)$ is the block success rate of $\mathrm{UE}_{\mathrm{n}}$, which is the function of $\operatorname{SINR}\left(\gamma_{n}\right)$ [9]. $P^{\Delta}=\left(P_{1}^{\Delta}, \ldots, P_{N}^{\Delta}\right)$ becomes the optimal solution $\mathbf{P}$, if it satisfies the constraint (12). Otherwise, since the utility function $U_{n}\left(\gamma_{n}\right)$ of $\mathrm{UE}_{n}$ is an increasing function, the upper bound of the transmission power will be the maximum value. Hence the optimal power $P_{n}^{*}$ is expressed as

$$
P_{n}^{*}=\left\{\begin{array}{l}
P_{n}^{\Delta} \quad \text { if } 0 \leq P_{n}^{\Delta} \leq P_{\max , U E} \\
P_{\max , U E} \quad \text { otherwise }
\end{array} .\right.
$$

\section{Performance Evaluation}

We consider a rectangular area of $50 \mathrm{~km} \times 50 \mathrm{~km}$ on the ground, which is serviced by seven aNBs. The aNBs are assumed to be installed on captive balloons of $2 \mathrm{~km}$ in height, and the UEs are randomly distributed in these traffic cells. The free path loss model is adopted for the air-ground radio link. The main system parameters are given in Table I [2]. Naturally, joint time-frequency-power resource allocation is only achievable, if accurate channel state information (CSI) is available. In the performance evaluation, we assume that each aNB can get perfect CSI for all of its UEs in each schedule period.

Four different types of service requirements are considered, which are VoIP, web-browsing, file-download and video, which are supported at data rates of $64 \mathrm{kbps}, 128 \mathrm{kbps}, 384 \mathrm{kbps}$ and $1024 \mathrm{kbps}$, respectively. Each UE randomly opts for one of the four services. In order to evaluate the attainable performance of the proposed algorithm, the well-established Max-C/I, RR, Max-min algorithm and the joint time-frequency-power allocation strategy algorithm, which was referred as JEEP in [1] are employed in the same scenarios. 
TABLE I Simulation Parameters

\begin{tabular}{|c|c|}
\hline Carrier frequency & $2.6 \mathrm{GHz}$ \\
\hline Bandwidth & $5,10,15,20 \mathrm{MHz}$ \\
\hline Maximum transmit power of aNBs & $43 \mathrm{dBm}$ \\
\hline Maximum transmit power of UEs & $23 \mathrm{dBm}$ \\
\hline Modulation & QPSK \\
\hline Coding & Turbo $1 / 2$ \\
\hline DL:UL ratio & $1: 1$ \\
\hline
\end{tabular}

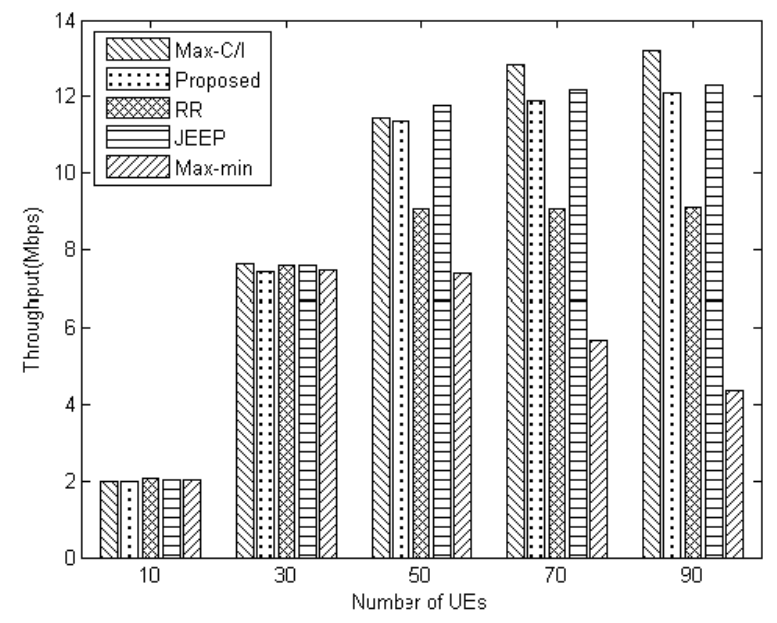

Figure 2. Throughput vs. the number of UEs

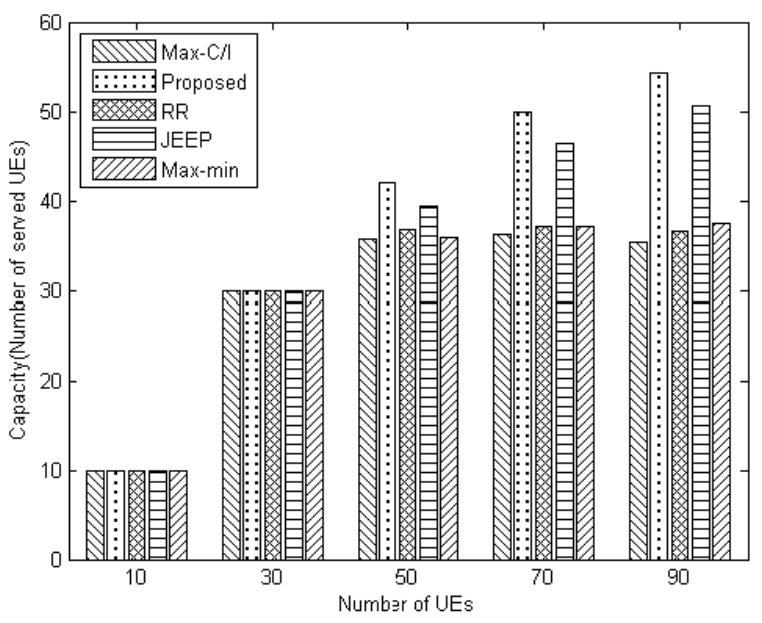

Figure 3. Capacity vs. the number of UEs

Figures 2, 3, and 4 show the system's throughput, capacity and fairness for different number of UEs respectively, where the channel bandwidth is fixed at $20 \mathrm{MHz}$. In the Max-C/I algorithm [13], in order to achieve the maximum data rate, aNBs will allocate each RB to the specific UEs having the best channel conditions. Hence, its throughput constitutes the upper bound of all the algorithms considered, as shown in Fig. 2 . However, its lower capacity and fairness is an obvious

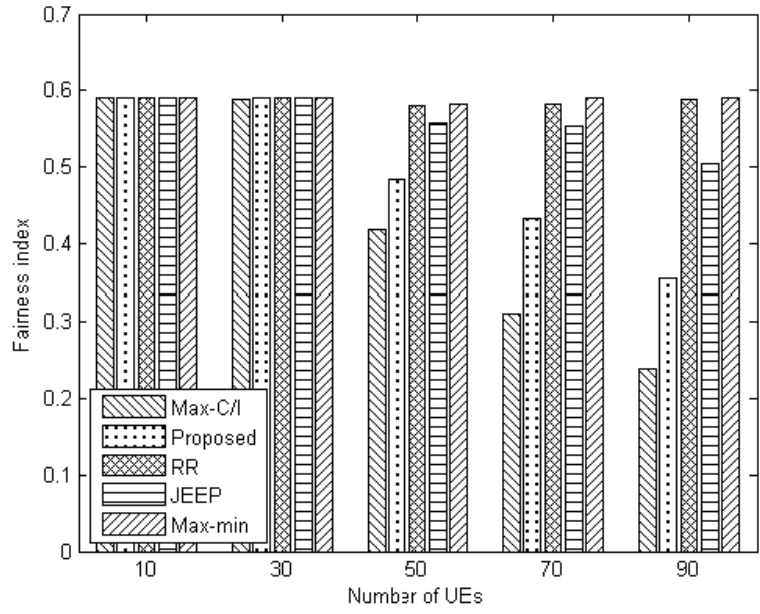

Figure 4. Fairness vs. the number of UEs

impediment, as shown in Fig. 3 and 4. Fairness is quantified in terms of the fairness index $\beta$ [12], which ranges between 0 and 1 , and UEs benefit from an increased fairness as $\beta$ approaches 1 .

The RR algorithm takes turns to allocate RBs to each UEs. Hence, its fairness constitutes the upper bound of all the algorithms, as shown in Fig. 4. The Max-min algorithm allocates more RBs to UEs suffering from degraded channel conditions to ensure fairness, but as a result, its system throughput becomes the lowest. However, it also achieves a good fairness.

In the JEEP algorithm [1], the resources are proportionally allocated according to each UE's channel conditions after satisfying the minimum requirement. Thus, the JEEP algorithm strikes a tradeoff between throughput and fairness, but, its capacity is significantly lower than that of the proposed algorithm, which achieves the highest capacity by sacrificing its throughput and fairness. In the proposed algorithm, the resources are allocated according to the novel system utility, more voice users are serviced. Thus its throughput and fairness become lower than that of JEEP.

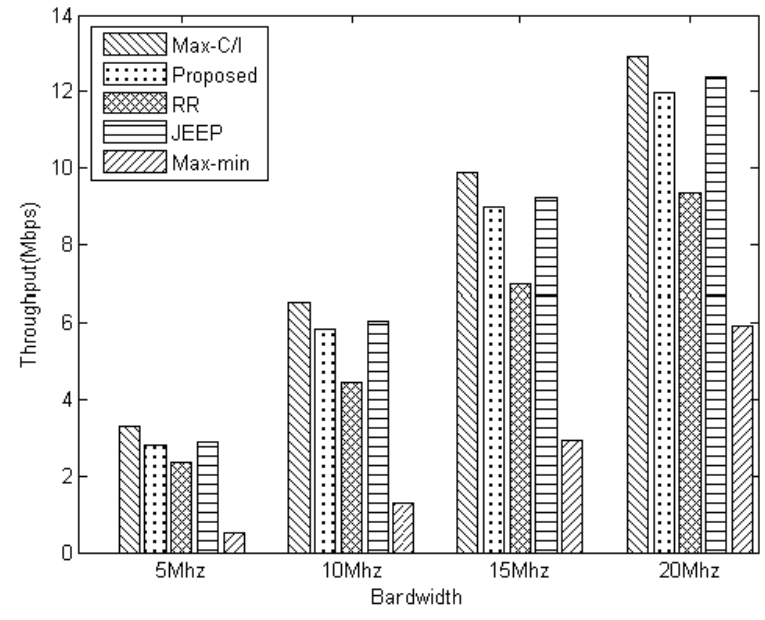

Figure 5. Throughput vs. channel bandwidth 


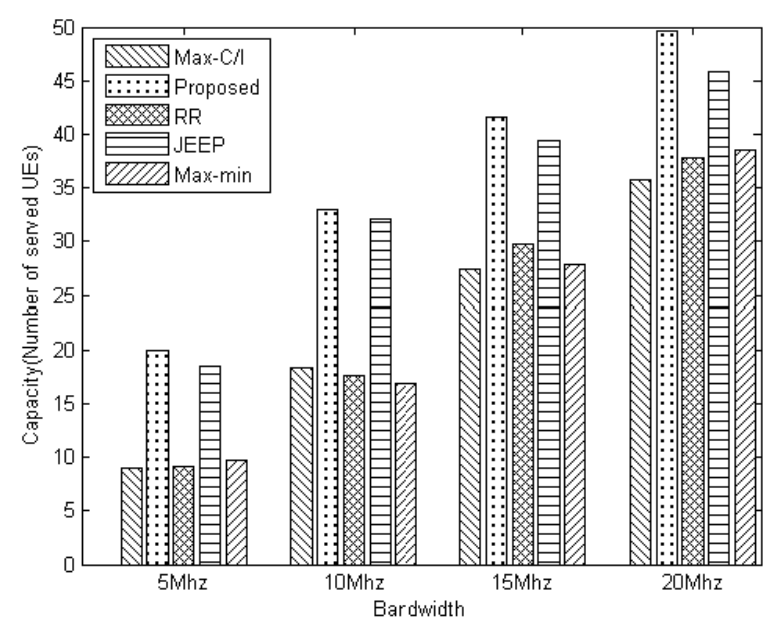

Figure 6. Capacity vs. channel bandwidth

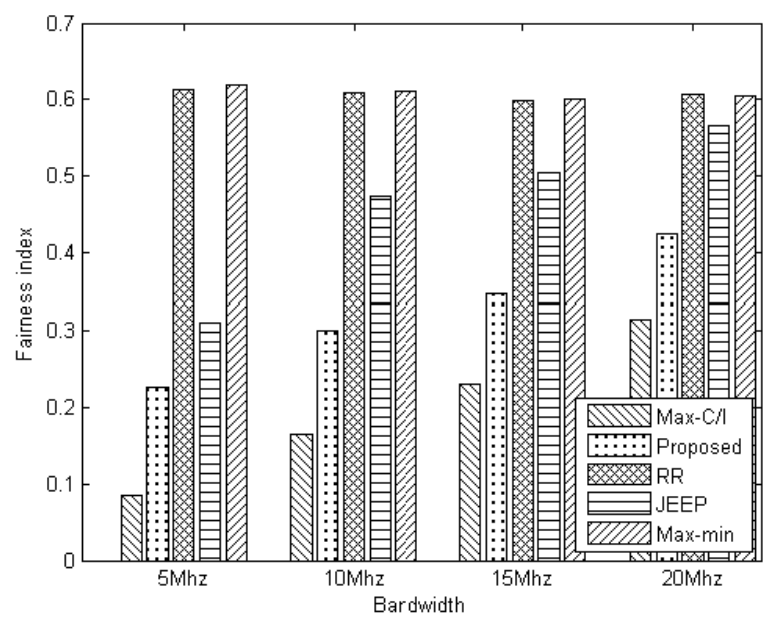

Figure 7. Fairness vs. channel bandwidth

Figures 5, 6 and 7 show the system's throughput, capacity and fairness for different channel bandwidths, respectively. In this scenario, 490 UEs are distributed in seven cells. Since the available resources become more abundant with the increase of bandwidth, the performance of each algorithm is improved. The Max-C/I algorithm attains the maximum throughput, while the RR algorithm and Max-min algorithm obtains the maximum fairness, and the proposed algorithm achieves the maximum capacity.

\section{CONCLUSIONS}

In this paper, an LMAP-based TD-LTE system was proposed for supporting emergency services in disaster areas. Game theory was used to develop a user-efficient RRM algorithm for both the uplink and downlink. Since in disasters people want to talk with their families, friends, etc, in order to confirm their safety, the most important requirement is to provide real-time voice communications with as many users as possible. The proposed algorithm adopted the ratio of the attainable data rate to the maximum required data rate as its salient utility function. Our simulation results demonstrated that the attainable number of serviced users of the proposed algorithm is significantly higher than that of all the other RRM algorithms. As a price, its throughput is lower than that of the Max-C/I and JEEP algorithms, and its fairness becomes lower than that of JEEP algorithms.

We are carrying out researches in the following three topics. Firstly, our proposed utility function is only the necessary condition of the maximum number of serviced users, not the sufficient condition. So we are designing a new utility, which is the necessary and sufficient condition of the maximum number of serviced users. Secondly, we are considering the sensitivity of the achievable performance to the imperfect CSI estimation. Thirdly, we are researching joint time-frequency-code-power resource algorithms for the LMAP-based TD-LTE system.

\section{REFERENCES}

[1] L. Zhao, Li Cong, F. Liu, "Joint time-frequency-power resource allocation for Low-medium altitude platforms based WiMAX Networks," IET Communications, May 2011, pp.967-972.

[2] 3GPP TS 36.211 V8.6.0, Physical Channels and Modulation (Release 8), Mar. 2009. [online]. Available:www.3gpp.org.

[3] L. Anchora, L.Canzian, L.Badia, M. Zorzi,"A characterization of resource allocation in LTE systems aimed at game theoretical approaches," IEEE International Workshop on CAMAD, 2010, pp.47-52.

[4] E. Yaacoub and Z. Dawy,"A game theoretical formulation for proportional fairness in LTE uplink scheduling," IEEE Wireless Commun. Netw. Conf., 2009, pp.1-5.

[5] G. Song, Y. Li, "Adaptive resource allocation based on utility optimization in OFDM,” IEEE GLOBECOM, Volume 2, Dec.2003, pp.586 - 590.

[6] W. Kuo, W. Liao, "Utility-based optimal resource allocation in wireless networks," IEEE GLOBECOM, Volume 6, Dec.2005, pp.5.

[7] C. Curescu, S. Nadjm-Tehrani, "Time-aware utility-based resource allocation in wireless networks," IEEE Transactions on Parallel and Distributed Systems, July 2005, pp.624-636.

[8] G. Song, Y. Li, "Utility-based resource allocation and scheduling in OFDM-based wireless broadband networks" IEEE Communications Magazine, Dec. 2005, pp.127 - 134.

[9] Z. Zhaoyang, S. Jing, C. Hsiao-Hwa, M. Guizani, Q. Peiliang, “A Cooperation Strategy Based on Nash Bargaining Solution in Cooperative Relay Networks," IEEE Transactions on Vehicular Technology, July 2008, pp. 2570-2577.

[10] V.Srivastava, J.Need, A. B. Mackenzie, "Using game theory to analyze wireless ad hoc networks," Commun. Surveys \& Tutorials, 2005, pp.46-53.

[11] S. Boyd, L. Vandenberghe, "Convex Optimization", Cambridge University Press, 2004, pp.1074-1079.

[12] E.L. Hahne, "Round-robin scheduling for max-min fairness in data networks," IEEE Journal on Selected Areas in Communications, Sep. 1991, pp.1024-1039.

[13] S. Periyalwar, B. Hashem, G. Senarath, K. Au, R. Matyas, "Future mobile broadband wireless networks: a radio resource management perspective," Wiley Journal of Wireless Communications and Mobile Computing, vol. 3, no. 7, Nov. 2003, pp. 803-816.

[14] F. Adachi, "Wireless Future," invited presentation for IEEE VTC-2011 Spring, May 2011, available at http://www.mobile.ecei.tohoku.ac.jp/. 